\title{
Ser adolescente e ser pai/mãe: Gravidez adolescente em uma amostra brasileira
}

\author{
Eva Diniz Bensaja dei Schiro \\ Silvia Helena Koller \\ Universidade Federal do Rio Grande do Sul
}

\begin{abstract}
Resumo
O estudo teve por objetivo investigar as características associadas à gravidez adolescente em uma amostra de adolescentes brasileiros de ambos os sexos. Estudo transversal $(N=226)$ com $64,2 \%$ dos participantes do sexo feminino. A média de idade dos participantes foi 18,86 anos $(D P=1,35)$. Os participantes responderam a um questionário que investigava aspetos como: (1) vida sexual; (2) sentimentos com relação à gravidez; (3) relações com escola; (4) trabalho; e (5) família. Não foram encontradas diferenças de sexo para idade de início da vida sexual nem para o número de gravidezes relatadas. Constatou-se, no entanto, que a gravidez gerou mais sentimentos de vergonha nas meninas $(p<0,01)$ e indicação de necessidade de buscar trabalho para os meninos $(p<0,001)$. Os meninos revelaram maior número de repetições de ano e de expulsões da escola em relação às meninas $(p<0,05)$. Esses resultados sugerem que gravidez adolescente toma diferentes significados para os adolescentes, em função do seu sexo.
\end{abstract}

Palavras-chave: gravidez; adolescência; parentalidade; sexo.

\begin{abstract}
Being adolescent and being father/mother: Teen pregnancy among a Brazilian sample. The aim of this study was to investigate characteristics associated to pregnancy in a Brazilian sample. A transversal design was developed $(N=226)$. From the sample, $64.2 \%$ were girls. The mean age of the participants was $16,86(S D$ $=1,35)$. A questionnaire about sexual life; feelings related to pregnancy; relationship with school, work and family was used. No sex differences were found on the age of sexual life debut and the number of related pregnancies. Although, the pregnancy brought more feelings of shame to girls $(p<0.01)$, and the boys informed more the need of work $(p<0.001)$. It was also observed that boys, compared with girls, evidence more grade repetition as well as school expulsion $(p<0.05)$. These results suggest that pregnancy during adolescence has diverse meanings according to the sex.
\end{abstract}

Keywords: pregnancy; adolescence; parenthood; gender.

\section{Resumen}

Ser adolescente y ser padre/madre: Embarazo adolescente en una muestra brasileña de muchachos y muchachos. El objetivo de este artículo consiste en investigar las características asociadas con la gestación en adolescentes en una muestra de jóvenes brasileños de los dos sexos. Estudio transversal $(N=226)$ con el 64,2\% de los participantes del sexo femenino. La edad promedio de los participantes fue de 18,86 años $(S D=1,35)$. Los participantes han contestado un cuestionario de examen de aspectos tales como: (1) vida sexual; (2) sentimientos sobre el embarazo; (3) relaciones con la escuela; (4) trabajo; y (5) familia. No hubo diferencias entre los sexos en lo que atañe a la edad de inicio de la actividad sexual o al número de embarazos reportados. Se constató, sin embargo, que el embarazo ha generado más sentimientos de vergüenza en las muchachas $(p<0,01)$, y, para los muchachos, ha generado la necesidad de buscar trabajo $(p<0,001)$. Los muchachos han revelado mayor número de repetición y expulsión escolar en comparación con las muchachas $(p<0,05)$. Estos resultados sugieren que el embarazo adolescente adquiere distintos significados para los jóvenes, en función del sexo.

Palabras clave: embarazo; adolescencia; parentalidad; sexo. 
I números estudos investigam as consequências da gravidez durante a adolescência para o desenvolvimento da mãe e do bebê (Benson, 2004; Carniel et al., 2006; Moore \& BrooksGunn, 2002). Não obstante, muitas destas pesquisas tendem a não incluir o pai (Levandowski, 2001). Perante esse fato considerouse importante investigar a forma como a gravidez durante a adolescência se repercute no desenvolvimento individual em adolescentes de ambos os sexos.

A gravidez durante a adolescência ocorre entre os 14-19 anos maternos (World Health Organization [WHO], 2002). Do total de partos que ocorrem no mundo, $10 \%$ são de adolescentes, enquanto no Brasil essa taxa corresponde a $18 \%$ dos partos registrados (WHO, 2006). A gravidez durante a adolescência é considerada um evento que poderá prejudicar o desenvolvimento da(o) adolescente, tanto do ponto de vista pessoal quanto profissional e, por isso, se considera um desafio exigente. A necessidade de conciliar a vivência da própria adolescência, considerada uma época de desafios e definições individuais, com o papel parental é apontada como a principal dificuldade (Dias \& Aquino, 2006; WHO, 2006). Assim, os adolescentes terão que lidar com tarefas duplas: as desenvolvimentais associadas ao seu período de vida e as do exercício da parentalidade (Esteves \& Menandro, 2005).

Outro aspecto associado à gravidez durante a adolescência é o contexto psicossocial em que tende a ocorrer, frequentemente associada à: pobreza, baixa escolaridade, desemprego ou emprego precário (Dias, 2009; Heilborn et al., 2002). Não obstante, é necessário considerar que a gravidez durante a adolescência não ocorre em um grupo homogêneo de pessoas, mas em contextos particulares com características específicas. Apesar disso, muitos desses aspetos tendem a não ser contemplados nas pesquisas que se detêm nos riscos associados à gravidez, isolando-as do contexto de desenvolvimento em que ocorrem, assim como das características desenvolvimentais da(o) adolescente prévias à gravidez (Duncan, 2007).

Os estudos sobre a gravidez tendem, ainda, a ser desenvolvidos na perspectiva dos elementos do sexo feminino e, frequentemente, ficam por conhecer as perspectivas dos elementos do sexo masculino (Abeche, Maurmann, Baptista, \& Capp, 2007; Levandowski, 2001). Essa lacuna torna-se ainda mais evidente no caso da gravidez durante a adolescência (Abeche et al., 2007; Duncan, 2007; Levandowski, 2001). Segundo Levandowski (2001) somente 2,9\% das produções relativas à gravidez durante a adolescência publicadas entre 1990-1999 incluíam informações sobre o pai. Esse resultado indicaria a baixa incidência de investigações sobre a temática, quando comparadas às da maternidade em geral. A falta de pesquisas que envolvam o elemento masculino poderia estar associada à tradição de desempenho dos próprios papéis parentais na sociedade: a mãe seria responsável pela educação e cuidado da criança, enquanto o pai desempenharia o papel de provedor da família (Levandowski, De Antoni, Koller, \& Piccinini, 2002). No entanto, é importante investigar o papel masculino na gravidez durante a adolescência, já que a presença paterna é apresentada como um dos fatores minimizadores de risco tanto para a mãe adolescente quanto para o bebê (Figueiredo, 2003; Jacard, Dodge, \& Dittus, 2003). Assim, a presença ou a ausência do pai, assim como o seu envolvimento na gravidez, são elementos que podem influenciar a forma como a gravidez é vivida e o tipo de relação estabelecida com a criança (Corkindale, Condon, Russell, \& Quinlivan, 2009).

A parentalidade durante a adolescência é descrita como uma tarefa exigente (Trindade \& Menandro, 2002). Não obstante, algumas características positivas são mencionadas, nomeadamente o aumento de responsabilidade, o amadurecimento face às responsabilidades e qualidade da relação com o(a) filho(a). Estas características tendem a ser comuns tanto para as meninas quanto para os meninos que se tornam pais (Corkindale et al., 2009; Esteves \& Menandro, 2005; Levandowski \& Piccinini, 2004). Não obstante, um conjunto de circunstâncias tende a associar-se ao aparecimento da gravidez durante a adolescência. As dificuldades escolares e o abandono precoce da escola são as características mais frequentemente citadas para a gravidez adolescente (Abeche et al., 2007; Almeida \& Hardy, 2007; Fagot, Pears, Capaldi, Crosby, \& Leve, 1998).

Em um estudo com 309 gestantes adolescentes brasileiras (Abeche et al., 2007) verificou-se que 55,7\% dos pais do bebê possuíam apenas o ensino fundamental (média de idade do parceiro foi 20 anos). Nesse estudo constatou-se que, no momento da gravidez, somente $25,2 \%$ dos parceiros estudavam, enquanto $71,2 \%$ trabalhavam. No mesmo sentido vão os resultados de uma pesquisa realizada com adolescentes dos Estados Unidos, em que os companheiros das adolescentes que engravidam tendiam a ter um abandono precoce da escola e provirem de um meio socioeconômico fragilizado (Fagot et al., 1998).

O abandono escolar é, também, uma característica comum às meninas que engravidam. Uma pesquisa realizada em três cidades brasileiras (Porto Alegre, São Paulo e Salvador) (Aquino et al., 2003) revelou que de 2.405 meninas, 42,1\% já tinham abandonado a escola antes de engravidarem, enquanto $17,3 \%$ interromperam os estudos na sequência da gravidez; embora $20,5 \%$ o tenha feito apenas temporariamente. Outro estudo realizado com 2.563 mães adolescentes de Campinas verificou que $87,6 \%$ das mães revelaram não trabalhar, dedicando-se aos cuidados do seu/sua filho(a) (Carniel et al., 2006).

O contexto de vulnerabilidade social, nomeadamente o absentismo escolar, tende a associar-se ao aparecimento da gravidez durante a adolescência. Nesse sentido, o objetivo do presente estudo foi investigar as características associadas à gravidez durante a adolescência em meninos e meninas que se tornaram pais, nomeadamente na relação com a escola, trabalho e família

\section{Método}

\section{Delineamento}

O presente estudo faz parte de um estudo maior que teve como objetivo investigar as características biopsicossociais de adolescentes e jovens brasileiros (Pesquisa Nacional sobre Fatores de Risco e Proteção da Juventude Brasileira de Koller, Cerqueira-Santos, Morais e Ribeiro, 2005 e Dell' Aglio e Koller, 2011). No presente estudo recorreu-se a um delineamento transversal exploratório-descritivo. 


\section{Participantes}

O banco de dados inicial era composto por 7.200 participantes, com idades entre os 14-24 anos. Deste banco selecionaram-se todos aqueles com idade igual ou inferior a 19 anos e que revelaram já ter tido experiência de gravidez $(n=226)$, o que correspondia a $8,6 \%$ do total de participantes inquiridos (Ver Tabela 1). A amostra contou com $145(64,2 \%)$ participantes do sexo feminino e $81(35,8 \%)$ do sexo masculino. A média de idades deste grupo foi de 16,86 anos $(D P=1,35)$. Todos os participantes provinham de um nível socioeconômico baixo e frequentavam escolas da rede pública de sete capitais (Porto Alegre, Recife, São Paulo, Belo Horizonte, Brasília, Campo Grande e Manaus (Ver Tabela 1) e três cidades brasileiras (Arcos, Minas Gerais; Presidente Prudente, São Paulo; e Maués, Amazonas (Ver Tabela 1).

Tabela 1

Distribuição de Participantes com Experiência de Gravidez por Cidade $(N=226)$

\begin{tabular}{lc}
\hline \multicolumn{1}{c}{ Cidade } & $\begin{array}{c}\text { Participantes com experiência de } \\
\text { gravidez }^{\mathbf{a}^{*}} \\
\mathbf{\%}(\boldsymbol{n})\end{array}$ \\
\hline Recife & $12,6(44)+$ \\
Porto Alegre & $8,7(38)$ \\
São Paulo & $9,7(32)$ \\
Campo Grande & $5,0(17)-$ \\
Amazonas & $16,2(17)+$ \\
Distrito Federal & $10,6(2,9)$ \\
Presidente Prudente & $6,3(24)$ \\
Arcos & $5,0(7)$ \\
Belo Horizonte & $6,8(18)$ \\
Total & $8,6(226)$ \\
\hline
\end{tabular}

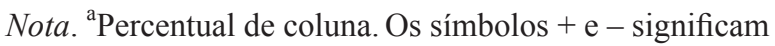
respectivamente um percentual de casos significativamente maior e menor que o esperado deslocados para a categoria (+: resíduo padronizado ajustado $+1,96$; - : resíduo padronizado ajustado $<-1,96)$.

\section{Instrumentos}

O questionário desenvolvido para a pesquisa Juventude Brasileira foi utilizado como instrumento de pesquisa e foi aplicado por uma equipe de pesquisadores e alunos de iniciação científica. Do questionário original, composto por 109 questões que investigava diversas dimensões da vida do(a) s adolescentes, selecionaram-se, para este estudo, aquelas relacionadas ao objetivo do estudo: dados sociodemográficos, aspectos da vida sexual (como por exemplo, idade da primeira relação sexual, tipo de parceiro na primeira relação, utilização de contracepção), da gravidez (idade da gravidez, número de gravidezes, avaliação subjetiva da gravidez, como emoções e sentimentos decorrentes da notícia de gravidez), relação com escola (número de anos de escolaridade frequentados, número de repetições de ano, por exemplo), trabalho (experiência laboral, tipo de vínculo empregatício foram algumas questões investigadas), identificação da principal figura sustentadora da casa e configuração familiar (por exemplo, com quem mora, satisfação com as relações familiares).

\section{Procedimento}

A pesquisa Juventude Brasileira teve como principal objetivo caracterizar a população adolescente/jovem de baixa renda do Brasil. Assim, recorreu-se à definição de nível socioeconômico baixo a partir dos cinco indicadores fornecidos pelo Instituto Brasileiro de Geografia e Estatística (UrbandataBrasil, 2002) como critério para a seleção das escolas de cada uma das cidades envolvidas na pesquisa: rendimento e nível de alfabetização do chefe da família, situação do domicílio (tipo de construção), existência de água encanada e rede de esgoto. Em São Paulo, utilizou-se também o Índice de Desenvolvimento Humano (IDH) de cada bairro disponibilizado pela Prefeitura da cidade. Foram listados os bairros que se encontravam abaixo de uma linha de corte, para, no mínimo, dois indicadores. A partir do número de bairros que foram selecionados, realizou-se uma amostra por conglomerados, tomando por critério o número de bairros e de escolas. A seleção das escolas foi feita segundo um sorteio a partir da lista de escolas (Municipais e Estaduais) em que se selecionou uma por bairro. De cada escola selecionada, sortearam-se turmas dos três turnos (manhã, tarde e noite) para participarem do estudo. Pretendeu-se a representatividade da população escolar, por isso turmas indicadas como "a melhor" ou a "pior" foram evitadas. Em algumas escolas, como os alunos da faixa etária pretendida estavam distribuídos por várias turmas, foram agrupados numa sala para a aplicação do questionário. O questionário foi aplicado em grupo, após a instrução geral para os alunos da sala e o seu preenchimento foi individual. O questionário foi aplicado por estudantes de pós-graduação em psicologia, auxiliados por estudantes de graduação da área.

Após o sorteio das escolas apresentaram-se os objetivos da pesquisa de forma a obter-se a autorização para a coleta de dados. Num momento posterior ao consentimento, os adolescentes/jovens foram convidados a participar da pesquisa. O objetivo da pesquisa foi explicado, assim como a garantia de confidencialidade e anonimato dos dados obtidos. Para aqueles que mostraram interesse em participar foi lido o Termo de Consentimento Livre e Esclarecido (TCLE) e enviada uma via para os responsáveis legais dos estudantes com menos de 18 anos. As questões éticas referentes à pesquisa foram asseguradas quanto à integridade dos participantes, conforme consta da Resolução $n^{\circ} 196 / 96$, que regulamenta a pesquisa com seres humanos. O projeto foi aprovado pelo Comité de Ética de uma instituição superior de ensino, com o protocolo n ${ }^{\circ}$ 2006/533.

\section{Resultados}

Com o objetivo de investigar as diferenças entre meninos e meninas com experiência de gravidez durante a adolescência, foi utilizado o teste Qui-quadrado e o $t$-student para investigar as diferenças de resposta dos dois sexos em relação a: aspetos da vida sexual (parceiro da primeira relação sexual, frequência de utilização de método contraceptivo e tipo de método utilizado, idade da primeira gravidez e número de gravidezes mencionadas); avaliação da gravidez; relação com a escola; 
trabalho; identificação da principal figura sustentadora da casa; e configuração familiar. Em seguida serão apresentados os resultados, de acordo com o sexo. Estabeleceu-se como nível de significância $p<0,05$.

Segundo os resultados obtidos verificou-se que, por um lado, os meninos descreveram menos a namorada como a parceira da primeira relação sexual (56,3\%), em comparação às participantes do sexo feminino $(84,6 \%)$. Por outro lado, os meninos tenderam a ter a sua iniciação sexual com uma amiga (32,5\% vs. $4.9 \%$ das respostas das meninas), $\chi^{2}(4, N=223)=$ $38,87, p<0,001$ (Ver Tabela 2).

Relativamente à utilização de contracepção, verificou-se que os meninos foram aqueles que mais revelaram a ausência de utilização de métodos contraceptivos $(17,7 \%)$, contra a proporção de meninas que deram esta resposta $(7,1 \%), \chi^{2}(2, N=219)=6,29$, $p<0,05$. Também se assistiu a presença de diferenças entre os sexos quanto ao principal método contraceptivo utilizado. Por um lado, os meninos, em comparação às meninas, revelaram utilizar menos a pílula como método contraceptivo ${ }^{1}(31,4 \%$ vs. $56,0 \%)$, $\chi^{2}(1, N=204)=11,10, p<0,01$. Por outro lado, registrou-se que o preservativo é um método mais utilizado pelos participantes do sexo masculino $(88,6 \%$ vs. $74,6 \%), \chi^{2}(1, N=204)=5,47, p<$ 0,05 (ver Tabela 2). Não se registraram diferenças significativas entre os sexos para o número de gravidezes vividas, nem para os episódios de aborto relatados. A idade em que ocorreu a gravidez também não foi diferente nos dois sexos.

Tabela 2

Características da Vida Sexual para Participantes do Sexo Masculino e Feminino $(N=226)^{\text {a }}$

\begin{tabular}{|c|c|c|c|c|}
\hline \multirow{2}{*}{ Categorias } & \multicolumn{4}{|c|}{ Sexo } \\
\hline & & Masculino \% (n) & Feminino \% $(n)$ & $p$ \\
\hline \multirow{5}{*}{ Parceiro da primeira relação sexual } & Namorado(a) & $56,3(45)-$ & $84,6(121)+$ & \multirow{5}{*}{$<0,001$} \\
\hline & Amigo(a) & $32,5(26)+$ & $4,9(7)-$ & \\
\hline & Marido/Esposa & $1,3(1)$ & $6,3(9)$ & \\
\hline & Parente & $6,3(5)$ & $1,4(2)$ & \\
\hline & Outro & $3,8(3)$ & $2,0(4)$ & \\
\hline \multirow{3}{*}{ Frequência de uso de contraceptivo } & Nunca & $17,7(14)+$ & $7,1(10)-$ & \multirow{3}{*}{$\mathbf{0 , 0 4}$} \\
\hline & Às vezes & $36,7(29)$ & $36,4(51)$ & \\
\hline & Sempre & $45,6(36)$ & $56,4(79)$ & \\
\hline \multirow{2}{*}{ Método utilizado ${ }^{\mathrm{b}}$} & Pílula & 31,4 (22)- & $56,0(75)+$ & $<0,001$ \\
\hline & Camisinha & $88,6(62)$ & $74,6(100)$ & 0,02 \\
\hline
\end{tabular}

Nota. ${ }^{\mathrm{a}}$ Percentual de coluna. Os símbolos $+\mathrm{e}-$ significam respectivamente um percentual de casos significativamente maior e menor que o esperado deslocados para a categoria ( + : resíduo padronizado ajustado $+1,96$; - : resíduo padronizado ajustado $<-1,96)$. ${ }^{\mathrm{b}}$ Resposta múltipla sobre o(s) método(s) contraceptivo(s) utilizado(s) na relação sexual.

No entanto, observaram-se diferenças entre os sexos na avaliação sobre a gravidez (Ver Tabela 3). As participantes do sexo feminino descreveram mais a gravidez como geradora de vergonha (26,5\%), em contraponto aos meninos $(10,8 \%$; $\left.\chi^{2}(2, N=139)=9,58, p<0,01\right)$. Os meninos descreveram, em maior proporção, a gravidez como um acontecimento gerador de maior preocupação $\left(75 \%, \chi^{2}(2, N=156)=7,91, p<0,05\right) \mathrm{e}$ de necessidade de trabalho $(36,1 \%$ vs. $7,3 \%), \chi^{2}(2, N=132)=$ $16,92, p<0,001$.

A relação com a escola apenas se revelou diferenciador entre os sexos relativamente ao número de repetições de ano e de expulsões sofridas (Ver Tabela 4). Os meninos revelaram ter tido mais de duas reprovações $(60,3 \%)$, em comparação às meninas $\left(28,6 \% ; \chi^{2}(1, N=208)=6,86, p<0,05\right)$. Também foram os meninos que apresentaram maior proporção de expulsões da escola $(61,1 \%$ vs. $33,2 \%), \chi^{2}(1, N=216)=4,54$, $p<0,05$. Contudo, constatou-se que nenhum dos sexos revelou permanecer mais na escola ou, pelo contrário, interromper mais a sua frequência, como consequência da gravidez. Da mesma forma, também não se registraram diferenças entre os sexos, na série e no turno frequentado (Ver Tabela 4).
De acordo com as respostas obtidas, constatou-se que os participantes do sexo masculino trabalharam mais no último ano $(60,3 \%)$ em proporção às participantes do sexo feminino $(42,8 \%), \chi^{2}(1, N=223)=6,21, p<0,05$ (ver Tabela 4). Contudo, não se verificaram diferenças entre os grupos na modalidade de trabalho com carteira assinada. Apesar disso, constatou-se que, em proporção, os meninos trabalham mais sem carteira assinada $(13,6 \%)$, em comparação às participantes do sexo feminino $(5,6 \%), \chi^{2}(1, N=225)=4,32, p<0,05$. Os adolescentes que não trabalharam e não procuraram trabalho também não diferiram quanto ao sexo. Não obstante, observou-se que os meninos desenvolveram mais trabalhos informais, como "fazer bicos" (32,1\%), em comparação à proporção de participantes do sexo feminino $(10,4 \%), \chi^{2}(1, N=225)=16,35, p<0,001$ (Ver Tabela 5).

Associado à relação com o trabalho está a principal figura sustentadora da casa (Ver Tabela 6), em que o companheiro foi descrito de forma mais frequente, pelas participantes do sexo feminino $(19,4 \%)$, em comparação à proporção de respostas obtidas pelos meninos $(3,8 \%), \chi^{2}(1, N=223)=10,46, p<$ 0,01 . Contudo, as respostas obtidas revelaram diferenças na 
Tabela 3

Avaliação da Gravidez para Participantes do Sexo Masculino e Feminino $(N=226)$

\begin{tabular}{lccc}
\hline & \multicolumn{2}{c}{ Sexo } & $p$ \\
& Masculino \% (n) & Feminino \% (n) & \\
\hline Importante & $70,8(34)$ & $77,0(87)$ & 0,4 \\
Desejada & $25,0(11)$ & $23,8(25)$ & 0,5 \\
Geradora de vergonha & $10,8(4)-$ & $26,5(27)+$ & $\mathbf{0 , 0 1}$ \\
Geradora de preocupação & $75,0(36)+$ & $51,9(56)-$ & $\mathbf{0 , 0 2}$ \\
Escondida & $41,0(16)$ & $31,4(32)$ & 0,5 \\
Geradora de orgulho & $42,5(17)$ & $46,0(46)$ & 0,8 \\
Geradora de desemprego & $0,0(0)$ & $7,4(7)$ & 0,2 \\
Geradora de casamento & $11,4(4)$ & $21,6(21)$ & 0,4 \\
Geradora de casamento forçado & $8,6(3)$ & $6,3(6)$ & 0,2 \\
Geradora de necessidade de trabalho & $36,1(13)+$ & $7,3(7)-$ & $<\mathbf{0 , 0 0 1}$ \\
Geradora de interrupção escolar & $5,7(2)$ & $9,9(10)$ & 0,8 \\
& & & \\
\hline
\end{tabular}

Nota. ${ }^{\text {a }}$ Percentual de coluna. Os símbolos $+\mathrm{e}-$ significam respectivamente um percentual de casos significativamente maior e menor que o esperado deslocados para a categoria (+: resíduo padronizado ajustado $+1,96$; - : resíduo padronizado ajustado $<-1,96)$.

Tabela 4

Relação com a Escola e Frequência Escolar entre os Participantes do Sexo Masculino e Feminino $(N=226)^{\mathrm{a}}$

\begin{tabular}{|c|c|c|c|c|}
\hline \multirow[b]{2}{*}{ Características } & & \multicolumn{2}{|c|}{ Sexo } & \multirow[b]{2}{*}{$p$} \\
\hline & & $\begin{array}{c}\text { Masculino } \\
\%(n)\end{array}$ & $\begin{array}{c}\text { Feminino } \\
\%(n)\end{array}$ & \\
\hline \multirow{2}{*}{ Relação com a escola } & Estuda & $93,8(76)$ & $97,9(140)$ & \multirow{2}{*}{0,1} \\
\hline & Parou de estudar & $6,2(5)$ & $2,1(3)$ & \\
\hline \multirow{7}{*}{ Série } & 1 & $28,2(20)$ & $21,6(30)$ & \multirow{7}{*}{$\mathbf{0 , 0 7}$} \\
\hline & 2 & $15,5(11)$ & $20,9(29)$ & \\
\hline & 3 & $15,5(11)$ & $24,5(34)$ & \\
\hline & 5 & $8,5(6)$ & $0,7(1)$ & \\
\hline & 6 & $4,2(3)$ & $6,5(9)$ & \\
\hline & 7 & $9,9(7)$ & $8,6(12)$ & \\
\hline & 8 & $16,9(12)$ & $13,7(19)$ & \\
\hline \multirow{3}{*}{ Turno } & Manhã & $33,3(25)$ & $33,1(47)$ & \multirow{4}{*}{0,7} \\
\hline & Tarde & $26,7(20)$ & $21,8(31)$ & \\
\hline & Noite & $40,0(30)$ & $45,1(64)$ & \\
\hline \multirow{3}{*}{ Reprovação } & Nenhuma & $21,8(17)-$ & $41,4(58)+$ & \\
\hline & Uma & $17,9(14)$ - & $30,0(42)+$ & \multirow[t]{2}{*}{0,01} \\
\hline & Duas ou mais & $60,3(47)+$ & $28,6(40)-$ & \\
\hline \multirow{2}{*}{ Expulsão } & Sim & $61,1(11)+$ & $33,2(136)$ - & \multirow{2}{*}{0,02} \\
\hline & Não & $38,9(7)-$ & $66,8(274)+$ & \\
\hline
\end{tabular}

Nota. ${ }^{\text {a }}$ Percentual de coluna. Os símbolos $+\mathrm{e}-$ significam respectivamente um percentual de casos significativamente maior e menor que o esperado deslocados para a categoria (+: resíduo padronizado ajustado $+1,96$; - : resíduo padronizado ajustado $<-1,96)$.

descrição sobre com quem o filho mora. Constatou-se que os filhos, proporcionalmente, moram mais com as mães $(35,2 \%)$ do que com os pais $(18,5 \%), \chi^{2}(1, N=226)=6,97, p<0,01$. As participantes do sexo feminino foram também aquelas que revelaram morar mais com o seu companheiro $(24,1 \%$ vs. $12,3 \%), \chi^{2}(1, N=226)=4,53, p<0,05$.

\section{Discussão}

Perante os resultados obtidos, constatou-se que a gravidez durante a adolescência, em muitos aspetos, tem características distintas para cada um dos sexos, nomeadamente no uso de contracepção, na avaliação da gravidez, na relação com a escola e o trabalho. Esse fato evidencia o quanto a gravidez tem diferentes origens e repercussões na vida dos adolescentes de acordo com o seu sexo.

Um aspeto diferenciador dos sexos foi o início da vida sexual. As meninas tenderam a ter a sua primeira relação sexual com o namorado, enquanto os meninos se iniciaram sexualmente com uma amiga. Atesta-se, assim, uma diferença 
Tabela 5

Relação com o Trabalho entre os Participantes do Sexo Masculino e Feminino $(N=226)^{\mathrm{a}}$

\begin{tabular}{|c|c|c|c|}
\hline \multirow[b]{2}{*}{ Características } & \multicolumn{2}{|c|}{ Sexo } & \multirow[b]{2}{*}{$p$} \\
\hline & $\begin{array}{c}\text { Masculino } \\
\%(n)\end{array}$ & $\begin{array}{c}\text { Feminino } \\
\%(n)\end{array}$ & \\
\hline Trabalha ou trabalhou no último ano & $60,3(47)+$ & $42,8(62)-$ & $\mathbf{0 , 0 1}$ \\
\hline Trabalha sem carteira assinada & $13,6(11)+$ & $5,6(8)-$ & $\mathbf{0 , 0 4}$ \\
\hline Trabalha com carteira assinada & $11,1(9)$ & $11,8(17)$ & 0,88 \\
\hline Não trabalha e está procurando & $49,4(40)$ & $48,6(70)$ & 0,9 \\
\hline Faz bicos & $32,1(26)+$ & $10,4(15)-$ & $<0,001$ \\
\hline Trabalha por conta própria & $9,9(8)$ & $3,5(5)$ & 0,06 \\
\hline
\end{tabular}

Nota. ${ }^{\text {a }}$ Percentual de coluna. Os símbolos $+\mathrm{e}-$ significam respectivamente um percentual de casos significativamente maior e menor que o esperado deslocados para a categoria (+: resíduo padronizado ajustado $+1,96$; - : resíduo padronizado ajustado $<-1,96)$.

Tabela 6

Principal Figura Sustentadora e Constituição do Agregado Familiar entre os Participantes do Sexo Masculino e Feminino $(N=226)^{\mathrm{a}}$

\begin{tabular}{|c|c|c|c|c|}
\hline \multirow{2}{*}{\multicolumn{2}{|c|}{ Características }} & \multicolumn{2}{|c|}{ Sexo } & \multirow{2}{*}{$p$} \\
\hline & & Masculino \% (n) & Feminino \% (n) & \\
\hline \multirow{4}{*}{ Quem sustenta a casa ${ }^{b}$} & $\mathrm{O}(\mathrm{a})$ adolescente & $15,2(12)$ & $11,9(17)$ & 0,5 \\
\hline & Pai & $47,5(38)$ & $42,7(61)$ & 0,5 \\
\hline & Mãe & $58,2(46)$ & $55,9(80)$ & 0,7 \\
\hline & Companheiro/a & $3,8(3)-$ & $19,4(28)+$ & $<0,001$ \\
\hline \multirow{6}{*}{ Com quem mora ${ }^{b}$} & Mãe & $77,8(63)$ & $80,7(117)$ & 0,6 \\
\hline & Pai & $45,0(36)$ & $40,7(59)$ & 0,5 \\
\hline & Madrasta & $3,7(3)$ & $2,1(3)$ & 0,4 \\
\hline & Padrasto & $8,6(7)$ & $8,3(12)$ & 0,9 \\
\hline & Filhos & $18,5(15)$ & $35,2(51)$ & 0,008 \\
\hline & Companheiro & $12,3(10)-$ & $24,1(35)+$ & 0,03 \\
\hline
\end{tabular}

Nota. ${ }^{\text {a }}$ Percentual de coluna. ${ }^{\mathrm{b}}$ Resposta múltipla. Os símbolos $+\mathrm{e}-$ significam respectivamente um percentual de casos significativamente maior e menor que o esperado deslocados para a categoria ( + : resíduo padronizado ajustado $+1,96$; - : resíduo padronizado ajustado $<-1,96)$.

no contexto de iniciação sexual. Possivelmente, essa diferença decorre dos padrões culturais associados ao início da vida sexual (Lemay, Cashman, Elfenbein, \& Felice, 2007). Discute-se que, culturalmente, tende a ser valorizado o desempenho sexual do menino e que, por isso, a sua iniciação sexual seria dissociada dos afetos e mais frequentemente ocorreria com uma amiga; enquanto as meninas tenderiam iniciar-se sexualmente em um contexto afetivo e, assim, o namorado tende a ser mais frequentemente citado (Lemay et al., 2007). Quanto aos padrões de utilização de contracepção constatou-se que os meninos tendem relatar mais a ausência do seu uso que as meninas. Essa diferença decorreria da perspectiva de que a menina é que teria a responsabilidade de evitar a gravidez (GRAVAD, 2006).

Os métodos contraceptivos mais utilizados pelos participantes foram a pílula e a camisinha. É descrito que a pílula tende a ser um método menos utilizado em adolescentes, o que não sucedeu neste estudo (Lemay et al., 2007). Discute-se que a adolescente tenderia a utilizar menos a pílula por a sua utilização revelar uma sexualidade que, frequentemente, é ocultada. No presente estudo, pelo contrário, verificou-se que a pílula é o método mais utilizado pelas adolescentes. Possivelmente, esse resultado associa-se ao fato de as adolescentes já terem tido uma experiência prévia de gravidez, o que facilitaria a utilização da pílula (Abeche et al., 2007; Lemay et al., 2007).

As percepções e sentimentos expressos pelos participantes relativos à gravidez foram por vezes, contraditórios. Ou seja, a gravidez é descrita como um episódio positivo, embora com vivências negativas, tal como relatado em outras pesquisas (Bigras \& Paquette, 2007; Breheny \& Stephens, 2007; Levandowski \& Piccinini, 2004). Não obstante, sobressaiu a avaliação da gravidez como um evento de vida positivo e importante, tanto para as meninas como para os meninos. Em alguns casos, a gravidez foi mesmo descrita como desejada. $\mathrm{O}$ desejo da gravidez, apesar da idade, poderá decorrer das poucas 
oportunidades de realização destes adolescentes que tendem a provir de contextos marcados pela precariedade psicossocial (Dias \& Aquino, 2006; Gomes, Speizer, Gomes, Oliveira, \& Moura, 2008). É discutido (Breheny \& Stephens, 2007; Moore $\&$ Brooks-Gunn, 2002) que o desejo da gravidez poderia surgir por dificuldades em áreas fundamentais na adolescência, como relações sociais e atividades escolares. Nesse sentido, a gravidez pode ser encarada como uma oportunidade de desenvolvimento e desenvolvimento de um papel na sociedade, que não existia anteriormente (Duncan, 2007; Persona, Shimo, \& Tarallo 2004).

Apesar de a gravidez tender a ser avaliada positivamente, registraram-se diferenças na sua avaliação de acordo com os sexos. Destaca-se a caracterização da gravidez como geradora de mais vergonha nas meninas, enquanto os meninos a descreveram como mais causadora de preocupação e de necessidade de trabalho. Essas diferentes percepções traduziriam o reflexo dos valores culturais e sociais, na forma como se vive um acontecimento individual (Bronfenbrenner, 2001/2005). Nas meninas, a gravidez parece ser encarada como algo "mais errado" e assim gerador de vergonha, possivelmente pela sua ocorrência desvelar uma sexualidade existente, mas nem sempre lícita, com exposição a relações sexualmente desprotegidas (Lemay et al., 2007). Esse sentimento é reforçado por se observar que algumas participantes revelaram ter escondido a sua gravidez. Provavelmente, para evitar reações negativas por parte da sua família (Duncan, 2007; Esteves \& Menandro, 2005). Os meninos, por sua vez, associam a gravidez ao aparecimento da necessidade de trabalho. Possivelmente, o trabalho surgiria como forma de assumirem a sua paternidade. Apesar de os resultados obtidos não permitirem identificar a motivação para o desenvolvimento de uma tarefa de trabalho, discute-se que essa atividade seria a forma de os meninos assumirem o seu papel parental, como provedor, assegurando a sobrevivência e bem-estar da sua família (Heilborn et al., 2002; Trindade \& Menandro, 2002).

Verificou-se, também, que o casamento surge como uma das consequências da gravidez, e alguns dos adolescentes mencionaram mesmo que foram obrigados a fazê-lo. Esse fato indicaria, também, a influência da cultura e dos contextos sociais no desenvolvimento individual (Bronfenbrenner, 2001/2005): a concepção de que a gravidez deve se inserir em uma relação de casal, mas também por se considerar que o casamento seria um marco formal de entrada na vida adulta (Galambos \& Martinez, 2007) e, por isso, necessário para o desempenho do papel de pai e de mãe.

Observou-se que apenas uma pequena parte do(a) $\mathrm{s}$ participantes informou ter interrompido a escola após a descoberta da gravidez. Esse dado poderia indicar que a maioria dos adolescentes já se encontrava fora do sistema escolar quando surgiu a gravidez (Breheny \& Stephens, 2007; Esteves $\&$ Menandro, 2005). No entanto, dado que a maioria do(a)s participantes da pesquisa foi contatado(a) em escolas, depreendese que muitos dele(a)s terão reingressado à escola. No entanto, ao fazerem-no, apresentaram um maior registro no turno da noite, o que poderia traduzir a concomitância do desempenho do papel parental com o de trabalho e estudo (Dias \& Aquino, 2006; GRAVAD, 2006). O reingresso à escola, também, poderia revelar o desejo de se desenvolverem pessoalmente (Breheny
\& Stephens, 2007; Duncan, 2007; Pantoja, 2003). Algumas adolescentes revelam que ter tido um filho(a) ajudou-as a compreender aquilo que queriam para as suas vidas e atuar de acordo com isso (Seamark \& Lings, 2004). As adolescentes desse estudo relataram que a preocupação com o futuro dos filhos e a necessidade de serem um exemplo para ele(a) as ajudou a desenvolver essa atitude. $\mathrm{O}$ reingresso na escola foi nomeado como um desses aspectos.

Não obstante, assistiu-se a um padrão distinto, entre meninos e meninas, na relação com a escola. Constatou-se que os meninos apresentam mais repetições de ano e maior número de expulsões. Por exemplo, do total de 18 participantes que revelaram terem sido expulsos da escola, 15 tinham tido experiência de gravidez e, destes, 11 eram meninos. Estudos realizados tanto em contexto brasileiro (Abeche et al., 2007) quanto internacional (Fagot et al., 1998) discutem que meninos com experiência de gravidez evidenciariam maiores dificuldades escolares. Assim, haveria uma relação entre a exposição a comportamento de maior risco e à gravidez. Este dado corroborou outros achados da literatura (Carniel et al., 2006; Heilborn et al., 2002; Persona et al., 2004), nos quais se descreve que a gravidez durante a adolescência tenderia a ocorrer nos adolescentes com menor inserção social e escolar e que procuram o trabalho, como alternativa à escola.

Esse dado explicaria porque os meninos mencionaram trabalhar mais. Contudo, verificou-se que o fazem de forma mais precária, em comparação às meninas: trabalham mais sem carteira assinada e mais em atividades informais, como o "fazer bicos". Apesar de o Estatuto da Criança do Adolescente (ECA) prever condições de trabalho protegidas para os menores de 16 anos, como o de jovem aprendiz, observou-se que os participantes desta amostra não beneficiam desses protocolos. Esse dado revelaria como a gravidez durante a adolescência tenderia a surgir em contextos de maior precariedade e vulnerabilidade social (Esteves \& Menandro, 2005; Moore \& Brooks-Gunn, 2002).

Uma vez que os participantes do sexo masculino revelaram trabalhar mais, justifica eles serem descritos como os principais provedores da casa. Estes dados confirmariam o fato de a mãe tender a ser descrita como a figura cuidadora do bebê, enquanto o pai seria o responsável pelo seu sustento (Levandowski et al., 2002). O fato de os adolescentes se juntarem e passarem a viver juntos traduziria a efetividade no desempenho do seu papel parental, nomeadamente na educação e sustento do seu filho (Dias \& Aquino, 2006).

\section{Conclusão}

$\mathrm{Na}$ realização da pesquisa sobressaiu a influência dos contextos de desenvolvimento, em que se destaca a vulnerabilidade dos seus participantes, no desempenho do papel parental. Por esse motivo, seria interessante investigar a gravidez adolescente em outros níveis sociais, de forma a entender como diferentes contextos socioeconômicos podem interferir no desenvolvimento de pais adolescentes. Além disso, no desenvolvimento deste estudo, identificaram-se algumas limitações associadas ao instrumento utilizado. Uma vez que a gravidez foi investigada de forma retroativa, naturalmente 
o relato desse acontecimento sofreu modificações decorrentes da passagem do tempo. Assim, acredita-se que os relatos da gravidez estariam mais associados às condições de vida de que se dispunha no momento em que os participantes responderam ao questionário do que daquelas que efetivamente possuíam quando engravidaram. Nesse sentido, teria sido importante conhecer as características de vida dos adolescentes anteriores à gravidez, para que se pudesse avaliar o seu impacto no decurso das suas vidas.

Observou-se que a percentagem de gravidezes registradas neste estudo foi menor do que as observadas em outras pesquisas realizadas no Brasil (Dias \& Aquino, 2006; Heilborn et al., 2002). Essa diferença poderá ser discutida com base no local em que foram coletados os dados - escola, já que a literatura aponta que muitos das adolescentes que engravidam já não a frequentariam (Breheny \& Stephens, 2007; Dias \& Aquino, 2006; Duncan, 2007). Não obstante, considerou-se pertinente a coleta de dados em escolas, precisamente para investigar a permanência na vida escolar após a gravidez. Sobressaiu uma maior proporção de meninos que, em meio escolar, relataram a experiência de gravidez, o que sugeriria que muitas das adolescentes abandonaram a escola na sequência da gravidez e maternidade (Almeida \& Hardy, 2007). Esse dado confirmaria que são as meninas os elementos mais prejudicados perante a gravidez adolescente (Lemay et al., 2007).

$\mathrm{O}$ presente estudo trouxe informações relevantes sobre as características da gravidez durante a adolescência, de acordo com o sexo. É importante a investigação da temática na população do sexo masculino, nomeadamente pela lacuna de estudos com essa população (Abeche et al., 2007; Duncan, 2007; Levandowski, 2001); mas também por se verificar que a gravidez toma diferentes configurações na vida de meninos e meninas, nomeadamente, na relação com a escola, trabalho e na avaliação da própria gravidez. A identificação dessas características e o reconhecimento das diferenças existentes para cada um dos sexos é importante para os agentes de saúde e de políticas públicas. Ou seja, é importante a caracterização dos adolescentes brasileiros que engravidam para que, efetivamente, se possa trabalhar na prevenção. Os achados obtidos são pertinentes já que revelam aspectos da vida desses adolescentes que, muitas vezes, são subestimados.

\section{Referências}

Abeche, A. M., Maurmann, C. B., Baptista, A. L., \& Capp, E. (2007). Aspectos socio-econômicos do parceiro da adolescente gestante. Revista do Hospital de Clínicas de Porto Alegre. 27(1), 5-9.

Almeida, A. F., \& Hardy, E. (2007). Vulnerabilidade de gênero para a paternidade em homens adolescentes. Revista de Saúde Pública, 41(4), 565-572.

Aquino, E. M. L., Heilborn, M. L., Knauth, D., Bozon, M., Almeida, M. C., Araujo, J., \& Menezes, G. (2003). Adolescência e reprodução no Brazil: A heterogeneidade dos perfis sociais. Cadernos de Saúde Pública, 19(2), 377-388.

Benson, J. M. (2004). After the Adolescent Pregnancy: Parents, Teens, and Families.

Child and Adolescent Social Work Journal, 21(5), 435-455.

Bigras, M., \& Paquette, D. (2007). Estudo pessoa-processo-contexto da qualidade das interações entre mãe-adolescente e seu bebê. Ciência \&Saúde Coletiva
12(5), 1167-1174

Breheny, M., \& Stephens, C. (2007). Individual responsibility and social constraint: The construction of adolescent motherhood in social scientific research. Culture, Health and Sexuality, 9(4), 333-346.

Bronfenbrenner, U. (2001/2005). The bioecological theory of human development. In U. Bronfenbrenner (Org.), Making human beings human: Bioecological perspectives on human development (pp. 3-15). Thousand Oaks, CA: Sage.

Carniel, E. F., Zanolli, M. L., Almeida, C. A., \& Morcillo, A. M. (2006). Características das mães adolescentes e de seus recém-nascidos e fatores de risco para a gravidez na adolescência em Campinas, SP, Brasil. Revista Brasileira de Saúde Materno-Infantil, 6(4), 419-426.

Corkindale, C. J., Condon, J. T., Russell, A., \& Quinlivan, J. A. (2009). Factors that adolescent males makes take into account in decisions about unplanned pregnancy. Journal of Adolescence, 32, 995-1008.

Dell'Aglio, D. D., \& Koller, S. H. (2011). Adolescência e Juventude: Vulnerabilidade e contextos de proteção. São Paulo: Casa do Psicólogo.

Dias, A. C. G. (2009). Análise das expectativas de jovens que vivenciaram a gravidez na juventude. In R. M. Libório \& S. H. Koller (Orgs.), Adolescência e juventude: Risco e proteção na realidade brasileira (pp. 155-183). São Paulo: Casa do Psicólogo.

Dias, A. B., \& Aquino, E. M. (2006). Maternidade e paternidade na adolescência: Algumas constatações em três cidades do Brasil. Cadernos de Saúde Pública, $22(7), 1447-1458$.

Duncan, S. (2007). What's the problem with teenage parents? And what's the problem with policy? Critical Social Policy, 27(3), 307-334.

Esteves, J. R., \& Menandro, P. R. M. (2005). Trajetórias de vida: Repercussões da maternidade adolescente na biografia de mulheres que viveram tal experiência. Estudos de Psicologia, 10(3), 363-370.

Fagot, B. I., Pears, K. C., Capaldi, D. M., Crosby, L., \& Leve, C. S. (1998). Becoming an adolescent father: Precursors and parenting. Developmental Psychology, 34(6), 1209-1219.

Figueiredo, B. (2003). Os primórdios da construção do próprio no contexto da interacção mãe-bebê. Psicologia: Teoria, Investigação e Prática, 2, 311-322.

Galambos, N. L., \& Martinez, M. L. (2007). Poised for emerging adulthood in Latin América: A pleasure for the privileged. Child Development Perspectives, 1(2), 109-114.

GRAVAD (2006). Pesquisa de Adolescentes no Brasil. Recuperado de www. portal.saude.gov.br

Gomes, K. R., Speizer, I. S., Gomes, F. M., Oliveira, D. D., \& Moura, L. N. (2008). Who are the pregnant adolescents in the poorest state capital of Brazil? Public Health Nursing, 25(4), 319-326.

Heilborn, M. L, Salem, T, Rohden, F., Brandão, E. Knauth, D., Víctora, C., .. Bozon, M. (2002). Aproximações socioantropológicas sobre a gravidez na adolescência. Horizontes Antropológicos, 8, 3-45. doi: 10.1590/S010471832002000100002

Jacard, J., Dodge, T., \& Dittus, P. (2003). Do adolescents want to avoid pregnancy? Attitudes toward pregnancy as predictors of pregnancy. Journal of Adolescent Health, 33(2), 79-83

Koller, S. H., Cerqueira-Santos, E., Morais, N. A., \& Ribeiro, J. (2005). Juventude brasileira. Relatório técnico para o Banco Mundial. Washington DC: World Bank.

Lemay, C. A., Cashman, S. B., Elfenbein, D. S., \& Felice, M. E. (2007). Adolescent mothers' attitudes toward contraceptive use before and after pregnancy. Journal of Paediatrics Adolescence Gynaecology, 20, 233-240.

Levandowski, D. C. (2001). Paternidade na adolescência: Uma breve revisão da literatura internacional. Estudos de Psicologia, 6(2), 195-209.

Levandowski, D. C., \& Piccinini, C. A. (2004). Paternidade na adolescência: Aspectos teóricos e empíricos, Revista Brasileira de Desenvolvimento Humano, 14(1), 51-67.

Levandowski, D. C., De Antoni, C., Koller, S. H., \& Piccinini, C. A. (2002). Paternidade na adolescência e os fatores de risco e de proteção para a violência na interação pais-criança. Interações, 7(13), 77-100. 
Moore, M. R., \& Brooks-Gunn, J. (2002). Handbook of parenting: Being and becoming a parent. Nova Iorque: Lawrence Erlbaum.

Pantoja, A. L. (2003). "Ser alguém na vida": Uma análise sócio-antropológica da gravidez/maternidade na adolescência, em Belém do Pará, Brasil. Cadernos de Saúde Pública, 19(2), 335-343.

Persona, L., Shimo, A. K. K., \& Tarallo, M. C. (2004). Perfil de adolescentes com repetição da gravidez atendidas num ambulatório de pré-natal. Revista Latino-americana de Enfermagem, 12(5), 745-750.

Seamark, C. J., \& Lings, P. (2004). Positive experiences of teenage motherhood:
A qualitative study. British Journal of General Practice, 54, 813-818.

Trindade, Z. A., \& Menandro, M. C. (2002). Pais adolescentes: Vivência e significado. Estudos de Psicologia (Natal), 7(1), 15-23.

Urbandata-Brasil (2002). Panorama editorial do Rio de Janeiro. Rio de Janeiro, $n^{\circ}$ 8, Censo 2000-IBGE. Rio de Janeiro: Laboratório de Políticas Públicas.

World Health Organization (WHO; 2002). Relatório. Recuperado de www. dianova.pt/index

World Health Organization (WHO; 2006). Pregnant adolescents. Recuperado de www.who.int.

Nota

1. A utilização da pílula ou camisinha pode ser indicada por ambos os sexos quando utilizado como método contraceptivo na relação

Eva Diniz Bensaja dei Schiro, doutoranda em Psicologia pela Universidade Federal do Rio Grande do Sul (UFRGS), mestre em Psicologia pela Universidade Federal do Rio Grande do Sul (UFRGS), é pesquisadora e doutoranda no Centro de Estudos Psicológicos (CEP-RUA), Universidade Federal do Rio Grande do Sul (UFRGS). Endereço para correspondência: Rua Cabral, 162, ap. 22, 90420-120 Rio Branco, Porto Alegre, RS. Telefones: (51)3557-5843 e (51)8147-5843. E-mail: eva.diniz@ufrgs.br Sílvia Helena Koller, doutora em Educação pela Pontifícia Universidade Católica do Rio Grande do Sul (PUCRS), é pesquisadora e coordenadora do Centro de Estudos Psicológicos (CEP-RUA) da Universidade Federal do Rio Grande do Sul (UFRGS). E-mail: Silvia.koller@gmail.com 